Original Paper http://ajol.info/index.php/ijbcs $\quad$ http://indexmedicus.afro.who.int

\title{
Relation entre le taux de réussite académique et l'alimentation et les conditions de vie, les qualités nécessaires à l'apprentissage, et la relation entre étudiants et enseignants à l'Université de Pala, Tchad
}

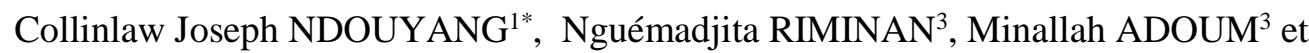 \\ Patrice DJIKOLOUM ${ }^{2}$
}

\author{
${ }^{l}$ Département des Sciences de Biologie, Faculté des Sciences Technique et Technologique, Université de Pala, \\ Route Minière de Baoré, BP 20 Pala, Tchad. \\ ${ }^{2}$ Département des Lettres Modernes, Faculté des Lettres, Arts et Sciences Humaines, Université de Pala, Route \\ Minière de Baoré, BP 20 Pala, Tchad. \\ ${ }^{3}$ Département de Géographie, Faculté des Lettres, Arts et Sciences Humaines, Université de Pala, Route \\ Minière de Baoré, BP 20 Pala, Tchad. \\ *Auteur correspondant; E-mail : ndouyang@yahoo.fr ; Tél.: (+235) 66080163.
}

\author{
Received: 09-08-2021 \\ Accepted: $15-12-2021$ \\ Published: 31-12-2021
}

\section{RESUME}

Les études universitaires au Tchad sont confrontées à des multiples difficultés. Ces difficultés entravent le succès académique, perturbent l'acquisition des connaissances et annihilent la performance. Cette étude vise à améliorer le taux de réussite par la maîtrise des facteurs d'échec académique. Ainsi, une étude sur les causes d'échec a été menée auprès des étudiants de licence 1 et 2 par un questionnaire. Les étudiants ayant pris part à l'enquête ont été soumis aux questions dans l'anonymat. Le dépouillement et les calculs de fréquences ont été effectués par Excel. Les classifications ascendantes hiérarchiques (CAH), les analyses en composantes principales (ACP), les tableaux de corrélation de Pearson ont été effectués par XLSTAT au seuil $\alpha=0,05$. Les réponses ont révélé que l'échec des étudiants en première année résultent d'une part des difficultés liées à l'alimentation, au déplacement ou transport, et d'autre part, au manque des qualités nécessaires à l'acquisition des connaissances et des compétences, au déficit de rapprochement entre enseignants et étudiants. Bien qu'il y ait de restrictions financières, les étudiants doivent lever les difficultés en matière d'organisation pour mieux se porter à l'aise et réussir dans leurs parcours universitaires. Des coopérations entre eux-mêmes et avec les enseignants leur sont nécessaires.

(C) 2021 International Formulae Group. All rights reserved.

Mots clés : Taux de réussite, alimentation, conditions, apprentissage, étudiants, enseignants.

\section{Relationship between academic success rate and food ingestion, life conditions, necessary qualities for learning, and relationship between students and teachers at the University of Pala, Tchad}

\begin{abstract}
Studies at universities in Tchad are faced to multiple difficulties. These difficulties prevent academic success, disturb learning and reduce excellence. This study aim at improving success rate by a management
\end{abstract}


against academic failure factors. In this way, a study about failure causes was lead towards students of licenses 1 and 2 by a survey. Students who have participated the survey have been submitted to questions without giving their names. Counting of survey sheets and calculation of frequencies were realized by Excel. The hierarchical ascendant classifications (HAC), the principal components analysis (PCA), Pearson correlation tables were purchased by XLSTAT at threshold $\alpha=0.05$. Results revealed that students failed to succeed at Level 1 and 2 bachelors, at one hand, difficulties are linked to food ingestion, to transport, and at other hand, to lack of necessaries qualities for learning and of bringing closer deficit between teachers and students. Although financial limitations exist, students must avoid difficulties by organizing easy plans in order to succeed at university. Cooperation between the very students and teachers are necessaries for improving success.

(C) 2021 International Formulae Group. All rights reserved.

Keywords: Success rate, food ingestion, conditions, learning, students, teachers.

\section{INTRODUCTION}

La réussite aux études fait la joie de l'apprenant et des siens, ensuite du pays. Mais au Tchad, la jeunesse subit les contrecoups des réformes successives et d'une modernisation brutale qui l'oppose aux institutions socioéducatives actuelles. On devra retenir que l'échec académique est l'un des facteurs qui déterminent le comportement de révolte des jeunes, frustrés par l'indifférence des adultes et l'injustice d'une société dont les lois tranchent avec les réalités que vivent ceux qui sont issus des familles démunies. En effet, la sousalimentation ou l'insuffisance de logement par exemple sont ressenties d'abord matériellement, mais aussi par la révolte, le dépit, une morne résignation ou cette honte secrète que les parents éprouvent devant les enfants et que ceux-ci ne tardent pas à partager, (Pinel, 2003; Paivandi, 2012). Cette frustration matérielle serait à l'origine des actes antisociaux (vol, crime) que commettent certains jeunes. D'après cet auteur, le système éducatif tchadien a été confronté à plusieurs situations défavorables pour son épanouissement pendant longtemps. Bien avant 1990, le caractère abstrait et élitiste de la formation, les taux excessifs de redoublement et d'abandon, le chômage et le sous-emploi des jeunes (malgré les besoins impérieux en cadres) constituaient l'une des contradictions majeures auxquelles le pays se trouvait confronté. Dans ce sens, la faillite de l'école tchadienne reconnue par les parents, les élèves et, surtout, les décideurs politiques, a connu des propositions et même des réformes qui, dans leur mise en œuvre ont fait fiasco, soit par manque de conviction (le cas en 1975), soit à cause d'une politique basée sur un pilotage à vue, et ce depuis 1979 à nos jours. Aujourd'hui, l'une des solutions sectorielles appliquée est le radicalisme ayant abouti à «l'abolition de la sélection scolaire » par la suppression des examens et concours de base, ainsi que la mauvaise gestion ou l'insuffisance des restaurations universitaires (Chupin, 2004 ; Levresse, 2009). Dès lors, l'effort consenti par l'étudiant en vue d'une bonne formation n'est plus pour lui une piste de réussite. Selon Laaser (2020), il existe une relation évidente entre la vie en société et son environnement aussi bien qu'entre la santé et l'environnement, attestant ainsi que la réussite dépend des moyens mis en œuvre. Sahouegnon et al. (2014) et Flenon et al. (2018) ont mis l'accent sur la qualité de l'alimentation chez les jeunes personnes en vue d'une bonne santé future chez l'adulte. Loukou et al. (2018) ont rapporté qu'il est important de ne pas négliger la nutrition minérale pour favoriser chez la personne humaine le développement physique et intellectuel.

Pratiquement, dans son parcours d'apprenant, l'élève devenu étudiant, dans ses cumuls de lacunes de connaissances intellectuelles, pense forcer même le succès après avoir quitté le chemin de ce même succès (Paivandi, 2012). Dans ces conditions, il est légitime de dire avec Banner et Cannon (2011a) qu'apprendre est une véritable aventure pour notre jeunesse. De ce fait, l'acquisition d'un savoir constitue-t-elle un bien en soi ? Selon ces auteurs, la compréhension est soutenue par un désir insatiable, la connaissance procure la simple 
joie et le savoir apporte une profonde satisfaction. La réussite scolaire ou académique ne nécessite pas seulement un bon environnement qui soit propice à l'apprenant. Des enseignants oublient souvent que l'enseignement est un art (Banner et Cannon, 2011b). Pour Bain (2011), la plupart des étudiants des meilleurs professeurs sont hautement satisfaits de l'enseignement reçu et incités par cet enseignement à poursuivre leurs études. Dans ce sens, Brauer (2011) retient les caractéristiques d'un enseignement universitaire de qualité soutenu par un bon rapport avec les étudiants, l'utilisation des méthodes pédagogiques qui facilitent l'apprentissage actif.

Aujourd'hui, dans les institutions d'enseignement supérieur, les taux d'abandon et d'échec en première année de licence sont élevés. A l'université de Pala, le taux d'abandon en première année de licence est en moyenne de $15,67 \%$, et les taux de réussite dans le même niveau oscillent entre $34,20 \%$ et $50,29 \%$, soit une moyenne de $42,25 \%$ pour les filières de Géographie et de Lettres Modernes. Cette étude avait pour objectif de rechercher donc les causes de ces échecs par une enquête menée auprès de 114 étudiants, sensiblement le triple de 30 , chiffre statistiquement admis dans un tel cas d'étude.

\section{MATERIEL ET METHODES \\ Méthodologie}

Pour mener à bien ce travail, le matériel utilisé dans cette étude a été constitué de deux éléments essentiels:

- la fiche d'enquête conçue pour un balayage effectif des causes possibles d'échec chez un étudiant ;

- le matériel biologique constitué d'étudiants auprès desquels l'enquête a été menée.

Sachant que E varie suivant la valeur de $\mathrm{P}$ et atteint son niveau maximum pour $P=0,5$ (CEFAÏ, 2003), la taille de l'échantillon $n$ a été déterminée par une probabilité $p$ de 0,5 pour une erreur inférieure ou égale à $5 \%$. L'erreur d'échantillonnage $\mathrm{E}$ est donnée par la formule ci-après :

$$
E=\sqrt{\frac{p(1-p)}{n}} \Longrightarrow n=\frac{p(1-p)}{E^{2}} .
$$

Les fiches d'enquête ont été mises à la disposition des Chefs de Départements de Géographie et de Lettres Modernes de la Faculté des Lettres, Arts et Sciences Humaines (FLASH) de l'Université de Pala à l'issue des résultats des examens académiques de l'année 2019/2020 en juin 2021. Les Chefs de Départements ont expliqué aux étudiants le bien-fondé de cette enquête pour eux-mêmes et pour l'entièreté de l'université. Les fiches ont été remplies par les étudiants ayant repris la licence 1 ou admis en licence 2 avec dette. Les étudiants exclus et réhabilités ont également pu donner leur point de vue sur les causes de leur échec. Ensuite, les Chefs de Départements des deux filières ont collecté les fiches remplies et les ont soumis aux dépouillements en vue des analyses statistiques. Pour ce faire, des conditions ont été fixées pour la détermination de la taille minimale de l'échantillon.

La fiche d'enquête sur les taux de réussite en GEO1 et LM1 comportait quatre principaux éléments qui sont (1) les provenances des étudiants, (2) les facteurs limitant ou facilitant la réussite académique, (3) les qualités nécessaires intrinsèques aux étudiants pour la réussite académique et (4) le contexte de relations humaines et sociales entre les étudiants et leurs environnements.

\section{Analyses statistiques}

Les calculs de fréquences ont été effectués par Excel. Les classifications ascendantes hiérarchiques (CAH), les analyses en composantes principales (ACP), les tableaux de corrélation de Pearson ont été effectués par XLSTAT au seuil $\alpha=0,05$.

\section{RESULTATS}

Les étudiants ajournés $(61,3 \%)$ et ceux admis avec dette $(32,1 \%)$ des niveaux de licence 1 et 2 ont donné l'essentiel de ces informations.

Les résultats de l'enquête sur les performances des étudiants en Géographie et Lettres Modernes de l'université de Pala sont présentés comme suit: La taille de 
l'échantillon a été de 114 étudiants repartis entre au moins 20 provenances ou lieux d'obtention du baccalauréat (Tableau 1). Il se dégage de ces résultats que la plupart des étudiants ayant pris part à cette évaluation sont originaires de Pala (43,0\%), suivis de ceux de Gounou-Gaya $(14,9 \%)$ et de Lagon $(13,2 \%)$. Les localités telles que Binder et Fianga ont été les moins représentées $(0,9 \%)$. Ces estimations renseigneraient sur la propension de ces localités pour l'université de Pala.

Le Tableau 2 présente les fréquences des réponses des étudiants sur leurs conditions de vie et d'étude. Il en ressort que la plupart d'entre eux vivent sans tuteur $(63,5 \%)$, ont faim tout le temps et en sont mêmes découragés (76\%), se plaignent de la distance séparant l'université du centre de la ville de Pala $(90,7 \%)$. Aussi, sont-ils préoccupés par le rythme des cours et des examens. Les uns trouvent que les cours sont très rapidement dispensés, tandis que les autres parlent de cours à vitesse moyenne. Néanmoins, les points de vue sont inversés quand il s'agit du déroulent des examens. Ces derniers sont dits très rapprochés $(71,8 \%)$ sinon bien espacés $(28,2 \%)$. La préoccupation est de savoir si les étudiants partagent cette fierté d'apprentissage des connaissances instinctuelles dans les deux départements. Malheureusement, la majorité reconnaît être là parce qu'elle ne savait où aller $(51,4 \%)$, suivie de ceux qui regrettent d'avoir choisi venir à l'université (32,4\%). Cette position des étudiants en rapport avec les conditions de vie et d'étude est donc une réalité à ne pas perdre de vue. Ces conditions ne manquent pas d'affecter les qualités nécessaires à l'apprentissage chez les étudiants.

Les qualités nécessaires ayant été examinées pour un étudiant sont présentées dans le Tableau 3. Ces résultats montrent que les étudiants viennent régulièrement aux cours $(87,3 \%)$ et sont contents de suivre leurs enseignants pendant le déroulement des séances $(60,0 \%)$, mais ils détestent certains cours $(30,0 \%)$. Ce qui est regrettable, c'est le fait que ceux qui ont de la fièvre ou ont des démangeaisons quand il y a un sujet qui exige de l'effort (exercice, sujet de travaux pratiques, contrôles continus des connaissances, examens terminaux...) ou qui ont de l'ennui quand l'emploi du temps est affiché, sont très nombreux $(78,4 \%)$. Cette démotivation pourrait à elle seule conduire à un échec à grande échelle. Beaucoup lisent uniquement leurs cours $(60,3 \%)$ sans diversification de lecture pour l'amélioration de leur base intellectuelle, pourtant ils aspirent à la qualité (96,5\%). Cette absence de curiosité intellectuelle est un mal pour nos étudiants. Leur imagination dont ils font preuve $(67,0 \%)$ ne semble pas aller ensemble avec leur réalité quotidienne en tant qu'apprenant. La vérité serait donc reconnue à ceux qui disent qu'ils ne savent pas imaginer $(25,7 \%)$, quand on sait que l'aspiration de la majorité est en désaccord avec la réalité de la réussite.

L'indiscipline et l'incivilité semblent caractériser les étudiants des deux départements. Nombre d'entre eux disent que les études sont difficiles $(55,9 \%)$ et d'autres ont préféré ne pas se donner de la peine pour les études (26,5\%). Ainsi, malgré qu'ils se joignent souvent aux autres pour comprendre (73,3\%), ils tombent dans le regret et optent pour la solitude en estimant qu'à seul on peut réussir ou qu'en groupe, le temps est perdu. L'incivilité dont ils font preuve en ne respectant point l'ordre $(59,3 \%)$ ou ne respectent pas les autres quand ils s'expriment $(28,4 \%)$ ou encore en ne respectant pas les normes ou principes, semble découler de leur conviction d'échec dans leur parcours académique.

Cette conviction s'expliquerait par cette tendance à reconnaître que tricher, c'est se faire du mal $(62,8 \%)$ tout en revenant tricher $(30,1 \%)$; ils refusent de prendre l'initiative $(73,8 \%)$ pour contribuer à créer leur propre «moi » $(26,1 \%)$. Désormais, le regard ne peut être tourné que vers le rapport entre les enseignants et les étudiants pour examiner les sources d'échec de ces derniers.

Dans cette étude, la relation entre les étudiants et les enseignants est rapporté dans le tableau de contexte (Tableau 4). Il en ressort que les étudiants dans leur ensemble $(75,7 \%)$ 
tirent du bénéfice de l'exigence de leurs enseignants pour le travail. Mais la relation de type amical entre les étudiants et les enseignants est faible $(28,7 \%)$ que celle de type non amical $(53,5 \%)$. Souvent cette dissension conduit à l'échec des apprenants. Ou bien, cette dissension peut être liée au fait que le choix d'une filière relève du hasard (22\%), ou au fait que la pléthore du niveau ait mis les étudiants en position d'échec à l'avance par le désordre que cela crée $(48,8 \%)$. Cette déception explique le déficit de lecture $(20,5 \%)$ bien que l'étudiant ait un programme de lecture bien monté $(66,1 \%)$.

Faute de bonnes relations entre enseignants et étudiants, comportement-clé pour la réussite des étudiants, ces derniers finissent par se retourner contre ce cadre d'étude qu'ils ont choisi pour bâtir leur vie future. C'est pourquoi, voyant leur attente déçue $(56,6 \%)$, ils estiment que les cours à l'université relèvent du désordre $(43,4 \%)$.

Tableau 1 : Lieu d'obtention du baccalauréat.

\begin{tabular}{|c|c|c|c|c|c|}
\hline & Totaux & $\begin{array}{c}\text { Géographie } \\
1,2\end{array}$ & $\begin{array}{c}\text { Lettres } \\
\text { Modernes } \\
\mathbf{1 , 2}\end{array}$ & $\begin{array}{l}\text { Fréquences } \\
\qquad(\%)\end{array}$ & $\begin{array}{c}\text { Ruraux+urbains } \\
(\%)\end{array}$ \\
\hline Provenances & 114 & 44 & 70 & 100,0 & 100,0 \\
\hline Binder rural & 0 & 0 & 0 & 0,0 & 0,9 \\
\hline Binder urbain & 1 & 1 & 0 & 0,9 & \\
\hline Fianga rural & 1 & 0 & 1 & 0,9 & 0,9 \\
\hline Fianga urbain & 0 & 0 & 0 & 0,0 & \\
\hline Gagal rural & 1 & 0 & 1 & 0,9 & 1,8 \\
\hline Gagal urbain & 1 & 1 & 0 & 0,9 & \\
\hline Gounou-Gaya rural & 11 & 3 & 8 & 9,6 & 14,9 \\
\hline Gounou-Gaya urbain & 6 & 1 & 5 & 5,3 & \\
\hline Lagon rural & 9 & 0 & 9 & 7,9 & 13,2 \\
\hline Lagon urbain & 6 & 1 & 5 & 5,3 & \\
\hline Léré rural & 2 & 0 & 2 & 1,8 & 5,3 \\
\hline Léré urbain & 4 & 0 & 4 & 3,5 & \\
\hline Pala rural & 25 & 8 & 17 & 21,9 & 43,0 \\
\hline Pala urbain & 24 & 14 & 10 & 21,1 & \\
\hline Torrock rural & 4 & 2 & 2 & 3,5 & 5,3 \\
\hline Torrock urbain & 2 & 1 & 1 & 1,8 & \\
\hline Kélo rural & 2 & 1 & 1 & 1,8 & 1,8 \\
\hline Kélo urbain & 0 & 0 & 0 & 0,0 & \\
\hline $\begin{array}{l}\text { Autre département rural } \\
\text { Autre département }\end{array}$ & 4 & 2 & 2 & 3,5 & 7,9 \\
\hline $\begin{array}{l}\text { urbain } \\
\text { Sans provenances }\end{array}$ & 5 & 3 & 2 & 4,4 & \\
\hline (mentionnées) & 6 & 6 & 0 & 5,3 & 5,3 \\
\hline
\end{tabular}


Tableau 2 : Facteurs limitant ou facilitant.

\begin{tabular}{|c|c|c|c|c|}
\hline Paramètres évalués & Totaux & $\begin{array}{l}\text { Géographie } \\
1,2\end{array}$ & $\begin{array}{l}\text { Lettres } \\
\text { Modernes } \\
1,2\end{array}$ & $\begin{array}{l}\text { Fréquences } \\
(\%)\end{array}$ \\
\hline a) Soutien à Pala : & 115 & 45 & 70 & 100,0 \\
\hline Vie avec un tuteur & 42 & 15 & 27 & 36,5 \\
\hline Vie sans un tuteur & 73 & 30 & 43 & 63,5 \\
\hline b) Alimentation : & 125 & 48 & 77 & 100 \\
\hline Je mange bien & 30 & 14 & 16 & 24,0 \\
\hline J'ai faim tout le temps & 53 & 19 & 34 & 42,4 \\
\hline Je suis découragé par la faim. & 42 & 15 & 27 & 33,6 \\
\hline c) Distance ville-UDP & 129 & 52 & 77 & 100 \\
\hline Je n'ai pas du temps pour lire & 12 & 5 & 7 & 9,3 \\
\hline Très grande distance & 48 & 20 & 28 & 37,2 \\
\hline La distance me fatigue & 69 & 27 & 42 & 53,5 \\
\hline d) Accélération des cours & 109 & 43 & 66 & 100,0 \\
\hline Cours très rapides & 35 & 15 & 20 & 32,1 \\
\hline Cours à vitesse moyenne & 53 & 17 & 36 & 48,6 \\
\hline Cours lents & 21 & 11 & 10 & 19,3 \\
\hline e) Séquences rapprochées des examens & 110 & 44 & 66 & 100 \\
\hline Examens très rapprochés & 79 & 36 & 43 & 71,8 \\
\hline Examens bien espacés & 31 & 8 & 23 & 28,2 \\
\hline Examens moins rapprochés & 0 & 0 & 0 & 0,0 \\
\hline f) Fourre-tout & 74 & 24 & 50 & 100 \\
\hline Je me suis trompé(e) de filière & 12 & 0 & 12 & 16,2 \\
\hline Si je savais, je ne venais pas à l'université & 24 & 9 & 15 & 32,4 \\
\hline Je ne savais où aller & 38 & 15 & 23 & 51,4 \\
\hline
\end{tabular}

Tableau 3 : Les qualités nécessaires intrinsèques.

\begin{tabular}{|c|c|c|c|c|}
\hline Paramètres évalués & Totaux & $\begin{array}{c}\text { Géographie } \\
1,2\end{array}$ & $\begin{array}{c}\text { Lettres Modernes } \\
1,2\end{array}$ & $\begin{array}{c}\text { Fréquences } \\
(\%)\end{array}$ \\
\hline 1) L'assiduité : & 118 & 46 & 72 & 100 \\
\hline Je viens souvent à l'école & 103 & 38 & 65 & 87,3 \\
\hline J'ai la paresse de suivre un enseignant & 4 & 1 & 3 & 3,4 \\
\hline Le cours n'est pas vérité. & 11 & 7 & 4 & 9,3 \\
\hline 2) L'enthousiasme : & 120 & 46 & 74 & 100 \\
\hline Je n'ai aucune joie à l'école & 12 & 2 & 10 & 10,0 \\
\hline Je suis content (e) de suivre un enseignant & 72 & 30 & 42 & 60,0 \\
\hline Je déteste certains cours & 36 & 14 & 22 & 30,0 \\
\hline 3) Le plaisir : & 79 & 34 & 45 & 100 \\
\hline Je n'ai aucun plaisir dans les études & 17 & 5 & 12 & 21,5 \\
\hline
\end{tabular}


J'ai de la fièvre ou des démangeaisons quand

il y a un sujet de travail (exercice, sujet de 28

travaux pratiques, $\mathrm{CC}, \mathrm{ET}$...)

J'ai de l'ennui quand l'emploi du temps est

$$
\text { affiché }
$$

4) La curiosité :

$$
34
$$

Je ne suis pas curieux

Je lis seulement mon cours

J'ai de la curiosité pour lire d'autres documents

C'est fatiguant de lire d'autres livres

Laissez-moi tranquille

5) L'aspiration :

Je veux devenir une personne de qualité

Non! Laissez-moi comme ça

4

Devenir «quelqu'un » pour faire quoi ?

J'imagine souvent ce qu'on m'enseigne

73

$\mathrm{CC}$ : contrôle continus ; ET : examens terminaux.

8

\begin{tabular}{|c|c|c|c|c|}
\hline 7) L'autodiscipline : & 102 & 39 & 63 & 100 \\
\hline Je ne me donne pas de la peine pour les études & 27 & 9 & 18 & 26,5 \\
\hline Les études sont difficiles & 57 & 23 & 34 & 55,9 \\
\hline Je n'ai pas la solitude pour réussir & 18 & 7 & 11 & 17,6 \\
\hline 8) La civilité : & 81 & 30 & 51 & 100 \\
\hline Il m'est difficile de respecter l'ordre & 48 & 21 & 27 & 59,3 \\
\hline J'aime arracher la parole aux gens & 23 & 6 & 17 & 28,4 \\
\hline Je ne respecte pas les normes ou principes & 10 & 3 & 7 & 12,3 \\
\hline 9) La coopération : & 120 & 47 & 73 & 100 \\
\hline Je me joins souvent aux autres pour comprendre & 88 & 33 & 55 & 73,3 \\
\hline A seul on peut réussir & 19 & 9 & 10 & 15,8 \\
\hline En groupe, le temps est perdu & 13 & 5 & 8 & 10,8 \\
\hline 10) L'honnêteté: & 113 & 44 & 69 & 100,0 \\
\hline Tricher, c'est se faire du mal & 71 & 26 & 45 & 62,8 \\
\hline Sans tricherie, pas de réussite & 8 & 3 & 5 & 7,1 \\
\hline Il faut tricher mais en secret & 34 & 15 & 19 & 30,1 \\
\hline 11) L'initiative : & 88 & 34 & 54 & 100 \\
\hline C'est un risque de prendre l'initiative & 48 & 22 & 26 & 54,5 \\
\hline Je ne peux pas contribuer à créer mon propre moi & 23 & 5 & 18 & 26,1 \\
\hline Je déteste prendre l'initiative & 17 & 7 & 10 & 19,3 \\
\hline
\end{tabular}

Tableau 3 : Les qualités nécessaires intrinsèques (suite et fin). 
Tableau 4 : Le contexte de relation étudiants/enseignants.

\begin{tabular}{|c|c|c|c|c|}
\hline Paramètres évalués & Totaux & $\begin{array}{l}\text { Géographie } \\
\qquad 1,2\end{array}$ & $\begin{array}{c}\text { Lettres } \\
\text { Modernes } \\
\mathbf{1 , 2}\end{array}$ & $\begin{array}{l}\text { Fréquences } \\
\qquad(\%)\end{array}$ \\
\hline \multicolumn{5}{|l|}{ 1) Qui sont vos enseignants? } \\
\hline $\begin{array}{r}\text { Tirez-vous du bénéfice de leur exigence pour } \\
\text { le travail? }\end{array}$ & 111 & 42 & 69 & 100,0 \\
\hline Oui & 84 & 38 & 46 & 75,7 \\
\hline Non & 13 & 4 & 9 & 11,7 \\
\hline Pas d'idées claires là-dessus & 14 & 0 & 14 & 12,6 \\
\hline $\begin{array}{l}\text { 2) Quel type de relation existe-il entre vous } \\
\text { et vos enseignants? }\end{array}$ & 101 & 38 & 63 & 100 \\
\hline Type amical & 29 & 14 & 15 & 28,7 \\
\hline Ambiguë & 18 & 5 & 13 & 17,8 \\
\hline Non amical & 54 & 19 & 35 & 53,5 \\
\hline 3) Ce que vous apprenez & 82 & 31 & 51 & 100 \\
\hline J'ai choisi la filière au hasard & 18 & 7 & 11 & 22,0 \\
\hline $\begin{array}{r}\text { C'est la pléthore du niveau qui m’a causé } \\
\text { l'échec }\end{array}$ & 40 & 16 & 24 & 48,8 \\
\hline $\begin{array}{r}\text { Ma personne serait mal formée pour ces cours } \\
\text { qu'on donne }\end{array}$ & 24 & 8 & 16 & 29,3 \\
\hline 4) Comment vous apprenez. & 112 & 43 & 69 & 100 \\
\hline J'ai un programme de lecture & 74 & 32 & 42 & 66,1 \\
\hline Je lis au hasard & 15 & 5 & 10 & 13,4 \\
\hline J'ai de problème pour lire & 23 & 6 & 17 & 20,5 \\
\hline 5) Du lycée à l'université & 113 & 42 & 71 & 100 \\
\hline Ce n'est pas comme au lycée & 64 & 27 & 37 & 56,6 \\
\hline C'est du désordre à l'université & 34 & 11 & 23 & 30,1 \\
\hline Cours mal donnés à l'université & 15 & 4 & 11 & 13,3 \\
\hline
\end{tabular}

\section{DISCUSSION}

Les étudiants ayant participé aux enquêtes sont de plusieurs provenances ou origines sociales. Le dendrogramme suivant (Figure 1) repartit les différentes provenances ou lieu d'obtention du baccalauréat en 7 classes selon le degré de similarité entre des réponses. Ainsi, on observe que 5 provenances (GounouGaya rural, Lagon rural, Lagon urbain, Léré urbain, Pala rural) forment une classe et 7 autres (Gounou-Gaya urbain, Léré rural, Pala urbain, Torrock rural, Kélo rural, autre département rural et autre département urbain) constituent une autre classe de réponses similaires. Ces réponses attestent d'un rapprochement étroit entre la qualité des formations scolaires et les milieux concernés dans une même classe. A partir de ces étudiants enquêtés, la difficulté de formation scolaire dans le Mayo-Kebbi Ouest est uniforme en milieu urbain que rural ; cela est vrai du moins pour les étudiants qui ont choisi de poursuivre leur cursus supérieur à l'université de Pala. Sinon, pourrait-on dire que les bacheliers de bon niveau scolaire ont déserté la région au profit d'autres institutions d'enseignements supérieurs. Cela pourrait s'expliquer en partie par le taux d'abandon élevé mentionné précédemment en première année de licence. Sans doute, des échecs viennent de l'abolition 
de la sélection scolaire et de la création des écoles par les autorités tchadiennes en charge de l'éducation nationale sans mesure d'accompagnement. La proposition recueillie avant 1990 et appliquée aujourd'hui n'a pas produit les effets escomptés (Chupin, 2004). Les internats et la restauration en milieu universitaire ne font pas partie des réalités académiques. En Europe, pour accroître la réussite des apprenants, les œuvres universitaires accordent des bourses ou subventions aux étudiants dont les provenances sont éloignées et le pouvoir d'achat faible (Martin, 2005 ; Vuarchere et al., 2006).

Il a été révélé que les inégalités sociales de réussite dans l'enseignement supérieur, la réussite d'un parcours de licence en trois ans n'est pas dépendante de la profession des parents, quand les autres facteurs sont contrôlés, notamment les caractéristiques scolaires (Morlaix et Suchaut, 2012). L'origine sociale conserve un impact sur une dimension plus « négative » par la sortie de l'enseignement supérieur sans diplôme. Les étudiants ayant une origine favorisée ont une meilleure probabilité de poursuivre leur cursus alors que les enfants d'ouvriers ont une probabilité d'abandon multipliée par 1,7 (Felouzis, 2000). C'est une situation d'inégalités sociales (Arzel et al., 2005). Lambert-Le Mener et Morlaix (2012) rapportent que la profession des parents, leur diplôme ou le revenu familial exercent une influence «incontestable » sur la réussite. Ces derniers arguent que les faibles effectifs d'étudiants défavorisés à l'université diminuent substantiellement la part de variance expliquée. Car l'échec est prégnant dans l'enseignement universitaire et principalement concentré en première année. Des étudiants des niveaux supérieurs ont la volonté d'obtenir de bonnes notes à l'ensemble des enseignements pouvant les pousser à sélectionner certaines matières et les répartir sur les deux sessions. Les étudiants de première année usent assez peu de ce type de stratégies. En outre, une déperdition en étudiants de bon niveau scolaire pourrait se situer dans le décalage de la rentrée académique.

Une autre cause de rendement académique médiocre aujourd'hui est constituée des facteurs limitant ou condition de vie. Tous les étudiants ajournés reconnaissent ici dans les études (Tableau 5) des difficultés statistiquement significatives venant de la faim ( $r \geq 0,812)$ et de la distance $(r \geq 0,833)$. Les difficultés dans les progressions des cours ou des examens ont été toutes statistiquement significatives $(r \geq 0,833)$. Les réponses des ajournés et des admis avec dette à ce sujet ont été significativement corrélées $(r \geq 0,722)$. Cela justifie la bonne position d'une bonne alimentation des étudiants dans la réussite académique.

La question de la faim, du transport et de la réussite universitaire a toujours préoccupé les étudiants sans ressources pour les études. Depuis que la bourse d'étude a été supprimée en 2016-2017 (Décret $N^{\circ}$ 686/2016), bon nombre de lauréats jugeait non opportun d'effectuer leurs études sur place au Tchad. Sur ce plan, le Cameroun constitue le pays d'accueil le plus sollicité.

Si la bourse a été supprimée en 20162017 chez les étudiants de l'intérieur, c'est que son projet de suppression a longtemps été maintenu en un document validé. Cet acte de suppression de la bourse aux étudiants a donné raison à Nietzsche qui a dit que «Bien des choses jugées bonnes par ce peuple étaient jugées par un autre comme honteuses et méprisables ». Cela explicite la raison fondée de la bourse aux étudiants. Car nos institutions d'enseignements supérieures ne sont pas valablement équipées en service sociaux tels que transport, soins par des centres hospitaliers universitaires (CHU), restaurants universitaires (Resto'U), bibliothèques, etc. La faim à elle seule est une barrière aux études quand on sait qu'elle est synonyme d'épuisement de l'organisme humain ou animal. La faim fait naître le désespoir chez l'apprenant et l'empêche de réfléchir, comme l'ont attesté les réponses à l'enquête. Suivant les plaintes des étudiants, ont été déplorées sur le campus de 
l'Université de Pala (UDP), l'irrégularité de la restauration, la monotonie des mets, la mauvaise hygiène de la préparation et de prise des repas. D'après les travaux de Ndouyang et al. (2020), les maladies métaboliques viennent surtout de la monotonie dans l'alimentation. Aussi, faut-il encourager la consommation des produits locaux sainement (BIMSA, 2009). Par conséquent, il faut rendre la restauration universitaire effective pour la santé, au lieu d'une simple tranche de filet de bœuf ou un tournedos non différents d'une alimentation banale et sans hygiène (Degnon et al., 2018 ; Djibrine et al., 2018). Pour Pavaut et al. (2011), l'alimentation doit être convenable en milieu universitaire. Selon Danton, «Après le pain, l'éducation est le premier besoin d'un peuple». Donc, pas de pain, pas d'éducation! Pourtant, la jeunesse est le seul bien qui vaille! Selon Karl Marx, «Si l'on ne crée que pour soimême [...], on ne deviendra jamais un homme épanoui, vraiment grand ». $\mathrm{La}$ formation universitaire est essentielle, mais une restauration digne et un transport adéquat des étudiants sont indispensables pour la santé (Delamaire, 2002 ; Peraya, 2011).

Il est reconnu combien importante la notion de "nourriture contre école". La discussion sur les classifications ascendantes hiérarchiques $(\mathrm{CAH})$ venait de montrer que les étudiants n'ont pas de spécificités de provenances, donc des individus sans ressources économiques particulières.

Enfin, les connaissances nutritionnelles ont montré que la faim est invalidante sur tous les plans. Elle est responsable de plusieurs maladies (Arzel et al., 2005), réduisant ainsi le rendement académique. Pour ce faire, une attention particulière doit être accordée au cadre des repas en vue de la sécurité alimentaire et de la qualité nutritionnelle de la restauration universitaire. Comme au Mali (Diarra et al., 2012) et en France (Lafay et al., 2002), des investigations sont nécessaires pour améliorer les restaurations universitaires au Tchad pour les rendre séduisantes et utiles. Un peu partout dans le monde, le seul mode de la performance est la comparaison de ce que l'on fait à ce qui se passe ailleurs (Kassel et Le Roux, 2020). Pour la Banque Mondiale, l'amélioration des conditions des études supérieures avec équité en Afrique subsaharienne doit s'élargir au-delà de l'élite. Cette amélioration doit prendre en compte les origines sociales en vue de l'intégration des étudiants démunis économiquement (Darvas et al., 2018). L'idéal est de réduire les effets et méfaits de la pauvreté et de la précarité sur le rendement intellectuel des étudiants d'origines sociales défavorables (Corinne, 2009). L'objectif de cette prise en charge est la réduction des inégalités sociales et l'amélioration de la santé au travers de l'alimentation. C'est ainsi qu'à Madagascar, l'alimentation scolaire a eu un effet très positif sur le court et le long terme pour les enfants qui en bénéficient, ainsi que des retombées sociales et économiques significatives à l'échelle nationale (WFP, 2019).

En plus des conditions d'étude qu'on doit réunir, il faut avoir des qualités nécessaires pour réussir dans ses études. Dans la majorité des cas, les corrélations de Pearson ( $r$ ) ont été statistiquement significatives suivant les réponses des "Ajournés" et des "Admis avec dette" (Tableau 6). Mais une observation se dégage aisément ici chez les exclus et les ajournés qui ont affirmé avoir eu la paresse de suivre un enseignant ou qui ont affirmé qu'à seul on peut réussir', Leurs réponses ont été fortement et positivement corrélées les unes aux autres $(\mathrm{r}=0,555$ à 0,$716 ; \mathrm{p}<0,05)$; les «Admis avec dette» ne disaient pas " avoir la paresse de suivre un enseignant'. Les corrélations statistiques significatives entre les réponses des «ajournés » et des «admis avec dette» montrent à suffisance la nécessité d'avoir des qualités nécessaires pour les études universitaires. Contrairement aux «Exclus», les «Ajournés » et les «Admis avec dette» partagent significativement les mêmes points de vue quant à ces qualités nécessaires $(\mathrm{r}=$ 0,722).

De ce qui précède, on peut dire que les étudiants ne sont pas sur la bonne piste pour réussir. C'est vrai, l'assiduité $(\mathrm{r}=0,852$ à 
$0,965)$ n'est pas la clé de tout. Le plus dur est d'étudier et d'apprendre seul. Lire seulement son cours est un effort insuffisant. Selon Banner et Cannon (2011b), c'est quand on pousse la recherche du savoir au-delà des limites strictement exigées que les fruits de l'assiduité sont particulièrement visibles. Et, l'apprentissage du savoir exige de l'organisation. Certains étudiants accusent leur biologie et se sous-estiment en disant : "Ma personne serait mal formée pour ces cours qu'on donne'. A l'assiduité s'ajoute l'enthousiasme. Il jaillit de l'intérêt que l'on prend aux choses. Mieux vaut donc travailler avec les enseignants qu'être contre eux. La misère des étudiants vient de ce qu'ils ignorent que l'acquisition du savoir exige des efforts, et que le plaisir dans les études ne vient pas du désordre. Cela nécessite donc de la curiosité pour rester ouvert à toutes les expériences afin d'être dirigé ensuite dans le bon chemin. Cependant, le manque de curiosité ne signifie pas que votre cerveau est desséché.

Mais à la curiosité doit s'ajouter l'aspiration plutôt qu'une ambition qui «est une sorte de folie» selon Baruch Spinoza. Parmi les bienfaits de l'aspiration, on note le bon usage de ce qu'on apprend. On pourra donc imaginer son futur radieux et le poursuivre comme objectif à atteindre. A l'instant même, l'imagination permet à l'étudiant de se considérer lui-même comme étudiant; elle permet de prendre intérêt et plaisir à l'étude. Ces derniers conduisent sans doute à l'autodiscipline, c'est-à-dire à considérer son propre bien-être d'étudiant et à s'imposer à soimême des critères exigeants. En rapport avec ses intérêts, l'étudiant doit retenir que la civilité gouverne les normes et les procédures qui assurent le fonctionnement de la société civilisée. Donc, la civilité sert son intérêt et le poussera à coopérer pour rendre plus facile l'apprentissage du savoir. Pour cela, il doit être honnête, éviter de faire ce qui est bien pour la mauvaise raison, donner de lui-même une image véridique. Comme l'ont rapporté Banner et Cannon (2011a), «L'honnêteté est la plus payante des manières d'étudier ».
Cette conviction pourra donc conduire l'étudiant à prendre de l'initiative lui permettant d'anticiper sur ce qui va suivre ou les étapes ultérieures de son programme de formation. Ainsi, pour réaliser ses aspirations, l'étudiant devra être partenaires de ses enseignants. Dans ces conditions (Paivandi, 2012), l'étudiant doit apprendre à apprendre afin d'apprendre sans se faire enseigner, se donner une culture langagière et une culture adéquate d'apprentissage, acquérir une méthodologie d'apprentissage.

Les piliers de l'apprentissage se composent des émotions qui sont les premiers piliers de l'apprentissage, et la motivation. Cette dernière assure que les apprenants acquièrent connaissances et compétences de manière pertinente. L'une des conditions de la motivation est que les apprenants se sentent capables de faire ce que l'on attend d'eux. A l'issue d'un colloque sur l'éducation, Marope et al. (2017) ont rapporté que le défi actuel en matière d'apprentissage et d'enseignement est de transformer les apprenants et l'apprentissage, donc éviter d'être calqué sur l'ancien modèle non participatif.

La relation entre étudiants et enseignants a des effets bénéfiques et multiples pour les étudiants. La conjugaison des efforts par les enseignants pour la réussite des étudiants n'est plus à démontrer. Dans le cas de cette étude (Tableau 7), les étudiants « ajournés » et les «admis avec dette» avaient de points de vue corrélés positivement et significativement aux variables $(r=0,506$ à $0,963 ; p<0,05)$. Parmi ces variables, celles à effets négatifs étaient donc défavorables aux étudiants, et celles à effets positifs leur ont été bénéfiques. Ainsi, les réponses telles que (i) absence de bénéfice pour l'exigence du travail, (ii) relation de type non amical, (iii) désordre à l'université, ou (iv) cours mal donnés sont autant des idées défavorables pour les étudiants. Selon Brauer (2011), le rapport avec l'enseignant est un des meilleurs prédicateurs de l'apprentissage des étudiants. Ces derniers recherchent chez leurs enseignants un certain nombre de qualités nécessaires. Les meilleurs 
enseignants devraient les posséder toutes, notamment le savoir, l'autorité, l'éthique, l'ordre, l'imagination, la compassion, la patience, la force de caractère et le plaisir (Banner et Cannon, 2011a). Mais ce que l'étudiant doit faire, c'est d'apprendre partout et en tout lieu avec plaisir. Il parvient ainsi à connaître la valeur du rapprochement enseignant/étudiant qui permet la connaissance essentielle ou des partenaires clairs. Pour cette raison, le type de relation entre enseignants et étudiants doit être amical. Cela permettra aux étudiants l'acquisition de l'autonomie. Celle-ci crée l'implication personnelle, la participation active, la prise de décisions à des différents niveaux, la mise en place de stratégies communicatives et cognitives (Pinel, 2003; Paivandi, 2012).

Pour réduire la distance entre étudiants et enseignants, ces derniers doivent laisser tomber l'hermine et la toge, ou tenter de descendre de leur estrade pour permettre une réalité sociale à l'origine d'un ensemble d'interactions, de transformations et de transactions entre des sujets agissants (Paivandi et Espinosa, 2013). Un bon enseignant communique à ses étudiants les conditions idéales de travail, notamment un esprit dégagé, un corps frais et dispos, un sujet d'étude intéressant, un environnement sympathique (Banner et Cannon, 2011a). Car les professeurs sont en réalité des missionnaires de leur matière (Banner et Cannon, 2011b). Les meilleurs enseignants ont une bonne connaissance de leur discipline; ce sont des chercheurs, artistes ou des savants actifs et accomplis (Bain, 2011). D'après les travaux d'un colloque sur l'éducation, Marope et al. (2017) ont rapporté que le défi actuel en matière d'enseignement est de transformer les apprenants et l'apprentissage, les enseignants et l'enseignement, l'évaluation, et le développement professionnel continu des enseignants. Il s'agit ici de rapprochement actif entre étudiants et enseignants.

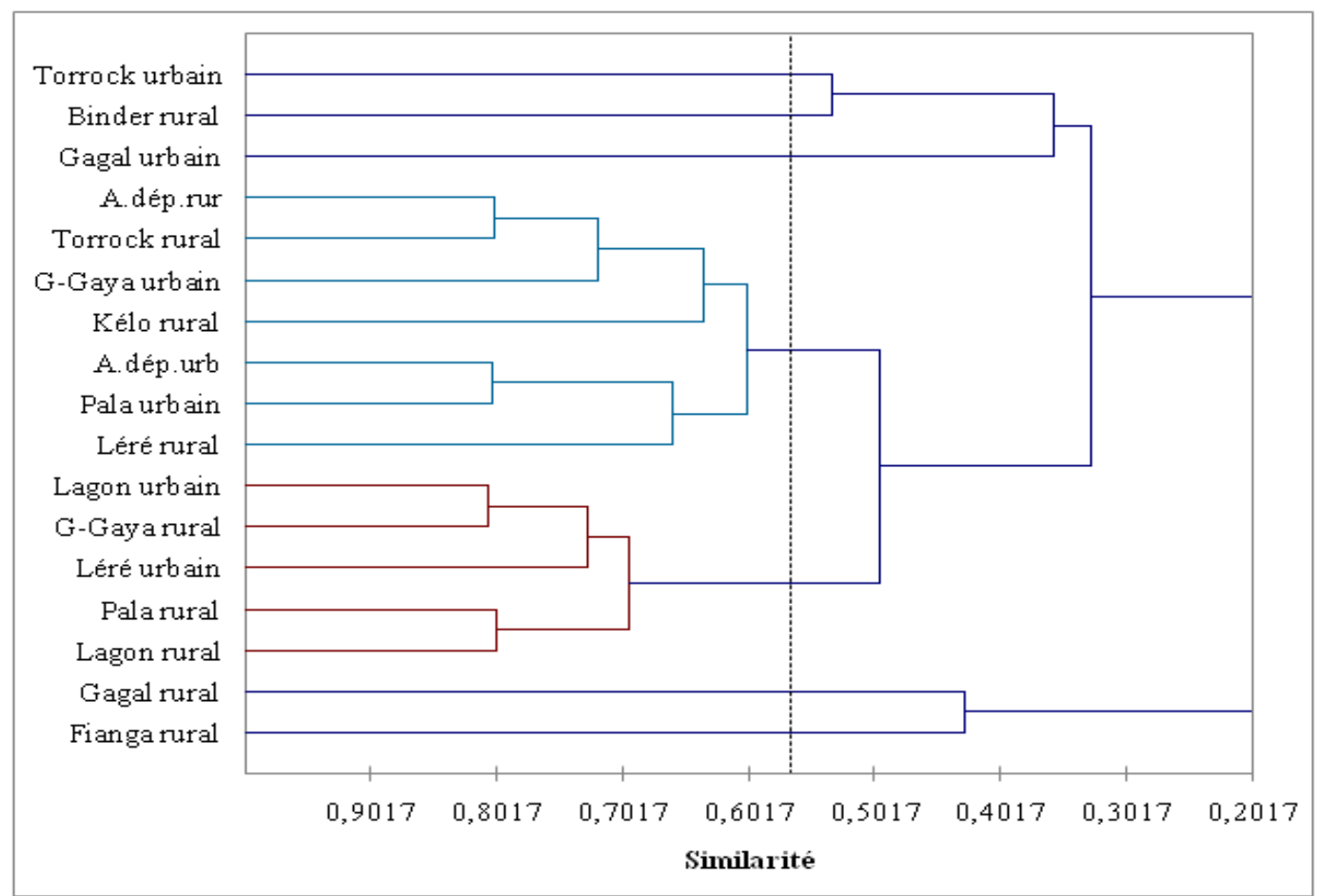

Figure 1 : Graphe de similarité des provenances des étudiants en Géographie et en Lettres Modernes (seuil : 0,$5691 ; 56,91 \%$ ).

A.dép.rural : Autre département rural ; A.dép.urb : Autre département urbain. 
Tableau 5 : Corrélations de Pearson des facteurs ou conditions d'étude.

\begin{tabular}{lrrr}
\hline & & \multicolumn{3}{c}{ Admis(e) avec } \\
Variables & Exclu(e) & Ajourné(e) & dette \\
\hline Vie avec un tuteur & 0,147 & $\mathbf{0 , 8 2 1}$ & $\mathbf{0 , 8 9 6}$ \\
Vie sans un tuteur & 0,403 & $\mathbf{0 , 9 5 8}$ & $\mathbf{0 , 7 2 8}$ \\
Je mange bien & 0,183 & $\mathbf{0 , 9 0 2}$ & $\mathbf{0 , 8 9 9}$ \\
J'ai faim tout le temps & 0,366 & $\mathbf{0 , 8 1 2}$ & $\mathbf{0 , 8 7 0}$ \\
Je suis découragé par la faim & 0,375 & $\mathbf{0 , 8 7 7}$ & 0,433 \\
Je n'ai pas du temps pour lire & 0,169 & $\mathbf{0 , 8 3 3}$ & $\mathbf{0 , 6 7 7}$ \\
Très grande distance & 0,340 & $\mathbf{0 , 9 4 8}$ & $\mathbf{0 , 7 5 4}$ \\
La distance me fatigue & 0,289 & $\mathbf{0 , 8 4 7}$ & $\mathbf{0 , 9 0 2}$ \\
Cours très rapides & 0,329 & $\mathbf{0 , 9 1 8}$ & $\mathbf{0 , 8 2 1}$ \\
Cours à vitesse moyenne & 0,308 & $\mathbf{0 , 9 7 4}$ & $\mathbf{0 , 7 7 5}$ \\
Cours lents & 0,174 & 0,482 & $\mathbf{0 , 7 4 4}$ \\
Examens très rapprochés & 0,295 & $\mathbf{0 , 9 2 9}$ & $\mathbf{0 , 8 9 7}$ \\
Examens biens espacés & 0,332 & $\mathbf{0 , 9 0 8}$ & $\mathbf{0 , 6 4 6}$ \\
Je me suis trompé(e) de filière & 0,398 & $\mathbf{0 , 8 3 3}$ & $\mathbf{0 , 7 7 3}$ \\
Si je savais, je ne venais pas à l'université & 0,184 & $\mathbf{0 , 8 7 3}$ & $\mathbf{0 , 8 0 5}$ \\
Je ne savais où aller & 0,320 & $\mathbf{0 , 9 5 4}$ & $\mathbf{0 , 7 2 1}$ \\
Exclu(e) & $\mathbf{1}$ & 0,256 & 0,270 \\
Ajourné(e) & 0,256 & $\mathbf{1}$ & $\mathbf{0 , 7 2 2}$ \\
Admis(e) avec dette & 0,270 & $\mathbf{0 , 7 2 2}$ & $\mathbf{1}$ \\
\hline \multicolumn{1}{c}{ Les valeurs en gras sont significativement différentes de 0 à un niveau de signification alpha= } & 05.05 \\
\end{tabular}

Tableau 6 : Corrélations de Pearson des qualités nécessaires.

\begin{tabular}{lccc}
\hline \multicolumn{1}{c}{ Variables } & Exclu(e) & $\begin{array}{c}\text { Ajour- } \\
\text { né(e) }\end{array}$ & $\begin{array}{c}\text { Admis(e) } \\
\text { avec dette }\end{array}$ \\
\hline Je viens souvent à l'école & 0,315 & $\mathbf{0 , 9 6 5}$ & $\mathbf{0 , 8 5 2}$ \\
J'ai la paresse de suivre un enseignant & $\mathbf{0 , 5 6 6}$ & $\mathbf{0 , 6 3 1}$ & 0,269 \\
Le cours n'est pas vérité. & 0,178 & $\mathbf{0 , 8 7 8}$ & $\mathbf{0 , 8 2 3}$ \\
Je n'ai aucune joie à l'école & 0,252 & $\mathbf{0 , 7 7 6}$ & $\mathbf{0 , 4 8 4}$ \\
Je suis content (e) de suivre un enseignant & 0,307 & $\mathbf{0 , 8 9 5}$ & $\mathbf{0 , 9 1 0}$ \\
Je déteste certains cours & 0,357 & $\mathbf{0 , 9 6 0}$ & $\mathbf{0 , 6 9 9}$ \\
Je n'ai aucun plaisir dans les études & 0,241 & $\mathbf{0 , 7 7 5}$ & $\mathbf{0 , 9 5 0}$ \\
J'ai de la fièvre ou des démangeaisons quand il y a & & & \\
un sujet de travail (exercice, sujet de travaux & 0,226 & $\mathbf{0 , 8 4 4}$ & $\mathbf{0 , 7 8 6}$ \\
pratiques, CC, ET...) & & & \\
J'ai de l'ennui quand l'emploi du temps est affiché & 0,189 & $\mathbf{0 , 9 4 7}$ & $\mathbf{0 , 6 7 4}$
\end{tabular}




\begin{tabular}{lccc} 
Je ne suis pas curieux & 0,433 & $\mathbf{0 , 6 6 5}$ & $\mathbf{0 , 5 6 4}$ \\
Je lis seulement mon cours & 0,448 & $\mathbf{0 , 9 5 7}$ & $\mathbf{0 , 7 8 0}$ \\
J'ai de la curiosité pour lire d'autres documents & 0,042 & $\mathbf{0 , 8 5 1}$ & $\mathbf{0 , 8 5 9}$ \\
C'est fatiguant de lire d'autres livres & $-0,042$ & $\mathbf{0 , 8 4 0}$ & $\mathbf{0 , 7 6 1}$ \\
Laissez-moi tranquille & 0,432 & $\mathbf{0 , 7 6 9}$ & $\mathbf{0 , 7 1 2}$ \\
Je veux devenir une personne de qualité & 0,307 & $\mathbf{0 , 9 7 0}$ & $\mathbf{0 , 8 5 8}$ \\
Non ! Laissez-moi comme ça & 0,030 & 0,432 & $\mathbf{0 , 8 0 3}$ \\
Devenir « quelqu'un » pour faire quoi ? & 0,387 & $\mathbf{0 , 6 7 8}$ & 0,355 \\
Je ne connais pas imaginer & 0,375 & $\mathbf{0 , 8 6 9}$ & $\mathbf{0 , 6 7 0}$ \\
J'imagine souvent ce qu'on m'enseigne & 0,208 & $\mathbf{0 , 9 5 7}$ & $\mathbf{0 , 8 4 1}$ \\
Inutile d'imaginer des scènes & 0,350 & $\mathbf{0 , 8 8 5}$ & $\mathbf{0 , 8 7 7}$ \\
Je ne me donne pas de la peine pour les études & 0,396 & $\mathbf{0 , 8 6 7}$ & $\mathbf{0 , 8 5 2}$ \\
Les études sont difficiles & 0,071 & $\mathbf{0 , 8 3 1}$ & $\mathbf{0 , 8 5 9}$ \\
Je n'ai pas la solitude pour réussir & 0,418 & $\mathbf{0 , 9 3 3}$ & $\mathbf{0 , 6 8 8}$ \\
Il m'est difficile de respecter l'ordre & 0,292 & $\mathbf{0 , 9 5 6}$ & $\mathbf{0 , 7 5 6}$ \\
J'aime arracher la parole aux gens & 0,050 & $\mathbf{0 , 7 3 7}$ & $\mathbf{0 , 7 0 1}$ \\
Je ne respecte pas les normes ou principes & 0,008 & $\mathbf{0 , 5 0 3}$ & $\mathbf{0 , 7 4 9}$ \\
Je me joins souvent aux autres pour comprendre & 0,346 & $\mathbf{0 , 9 7 4}$ & $\mathbf{0 , 7 7 3}$ \\
A seul on peut réussir & $\mathbf{0 , 5 5 5}$ & $\mathbf{0 , 7 1 6}$ & $\mathbf{0 , 7 4 9}$ \\
En groupe, le temps est perdu & $-0,041$ & $\mathbf{0 , 7 1 4}$ & $\mathbf{0 , 9 0 5}$ \\
Tricher, c'est se faire du mal & 0,372 & $\mathbf{0 , 9 0 0}$ & $\mathbf{0 , 8 6 8}$ \\
Sans tricherie, pas de réussite & $-0,047$ & $\mathbf{0 , 6 6 1}$ & $\mathbf{0 , 7 6 7}$ \\
Il faut tricher mais en secret & 0,206 & $\mathbf{0 , 8 8 8}$ & $\mathbf{0 , 6 3 0}$ \\
C'est un risque de prendre l'initiative & 0,192 & $\mathbf{0 , 8 9 8}$ & $\mathbf{0 , 8 8 3}$ \\
Je ne peux pas contribuer à créer mon propre moi & 0,278 & $\mathbf{0 , 8 3 3}$ & $\mathbf{0 , 6 6 5}$ \\
Je déteste prendre l'initiative & 0,266 & $\mathbf{0 , 9 1 0}$ & $\mathbf{0 , 7 5 6}$ \\
Exclu(e) & $\mathbf{1}$ & 0,256 & 0,270 \\
Ajourné(e) & 0,256 & $\mathbf{1}$ & $\mathbf{0 , 7 2 2}$ \\
Admis(e) avec dette & 0,270 & $\mathbf{0 , 7 2 2}$ & $\mathbf{1}$ \\
\hline Les valeurs en gras sont significativement différentes de 0 à un niveau de signification alpha= $0,05$. & \\
CC : contrôles continus ; ET : examens terminaux. & & &
\end{tabular}

Tableau 7 : Corrélations de Pearson des effets de relation entre étudiants et enseignants.

\begin{tabular}{lccc}
\hline \multicolumn{1}{c}{ Variables } & Exclu(e) & Ajourné(e) & $\begin{array}{c}\text { Admis(e) } \\
\text { avec dette }\end{array}$ \\
\hline Bénéfice de l'exigence pour le travail : Oui & 0,325 & $\mathbf{0 , 9 6 3}$ & $\mathbf{0 , 8 3 3}$ \\
Bénéfice de l'exigence pour le travail: Non & 0,014 & $\mathbf{0 , 7 2 1}$ & $\mathbf{0 , 8 7 9}$ \\
Pas d'idées claires là-dessus & 0,221 & $\mathbf{0 , 8 8 7}$ & $\mathbf{0 , 6 0 2}$ \\
Relation de Type amical & 0,405 & $\mathbf{0 , 8 7 6}$ & $\mathbf{0 , 7 9 7}$ \\
Relation de Type Ambiguë & 0,060 & $\mathbf{0 , 7 6 8}$ & $\mathbf{0 , 7 6 4}$
\end{tabular}




\begin{tabular}{lccc} 
Relation de Type Non amical & 0,219 & $\mathbf{0 , 9 2 9}$ & $\mathbf{0 , 8 0 5}$ \\
J'ai choisi la filière au hasard & $-0,098$ & $\mathbf{0 , 7 6 8}$ & $\mathbf{0 , 8 5 5}$ \\
C'est la pléthore du niveau qui m'a causé & 0,220 & $\mathbf{0 , 9 5 3}$ & $\mathbf{0 , 7 7 0}$ \\
l'échec & & & \\
Ma personne serait mal formée pour ces cours & 0,480 & $\mathbf{0 , 9 1 4}$ & $\mathbf{0 , 6 7 9}$ \\
qu'on donne & 0,416 & $\mathbf{0 , 9 2 9}$ & $\mathbf{0 , 8 3 6}$ \\
J'ai un programme de lecture & 0,233 & $\mathbf{0 , 8 2 3}$ & $\mathbf{0 , 7 8 9}$ \\
Je lis au hasard & $-0,092$ & $\mathbf{0 , 7 8 2}$ & $\mathbf{0 , 7 8 9}$ \\
J'ai de problème pour lire & 0,355 & $\mathbf{0 , 9 6 1}$ & $\mathbf{0 , 8 0 9}$ \\
Ce n'est pas comme au lycée & 0,102 & $\mathbf{0 , 8 4 0}$ & $\mathbf{0 , 9 2 4}$ \\
C'est du désordre à l'université & 0,433 & $\mathbf{0 , 9 1 4}$ & $\mathbf{0 , 5 0 6}$ \\
Cours mal donnés à l'université & $\mathbf{1}$ & 0,256 & 0,270 \\
Exclu(e) & 0,256 & $\mathbf{1}$ & $\mathbf{0 , 7 2 2}$ \\
Ajourné(e) & 0,270 & $\mathbf{0 , 7 2 2}$ & $\mathbf{1}$ \\
Admis(e) avec dette & \multicolumn{1}{c}{ Les valeurs en gras sont significativement différentes de 0 à un niveau de signification alpha $=0,05}$.
\end{tabular}

\section{Conclusion}

Pour rehausser le rendement académique d'étudiants, il faut repenser le soutien financier en faveur des origines sociales des démunis et faciliter les déplacements des étudiants dans leur ensemble. Aussi, faut-il une mentalité renouvelée des étudiants en vue des qualités nécessaires à l'acquisition des connaissances et des compétences académiques. Enfin, étudiants et enseignants doivent avoir le partage des connaissances dans des relations amicales et dignes de respect. Des études similaires ultérieures sont nécessaires pour rendre plus performante cette piste de connaissances des échecs académiques et leur maîtrise à l'université de Pala.

\section{CONFLIT D'INTERETS}

Les auteurs du présent article déclarent n'avoir aucun conflit d'intérêts.

\section{CONTRIBUTIONS DES AUTEURS}

CJN a initié le projet de publication sur le sujet, formulé la fiche d'enquête et assuré la rédaction. $\mathrm{PD}$ et $\mathrm{MA}$ ont collecté les données d'auprès des étudiants et NR a examiné le travail. Tous les auteurs ont relu tout le document.

\section{REMERCIEMENTS}

Nous exprimons nos reconnaissances aux Chefs de Département pour avoir conduit l'enquête auprès de leurs étudiants en vue de sa réussite.

\section{REFERENCES}

Arzel B, Golay M, Zesiger V, Kabengele Mpinga E, Chastonay P. 2005. Malnutrition et inégalités sociales. Bulletin des Médecins Suisses, 86(18): 1093-1096.

Bain K. 2011. Les Secrets des Meilleurs Professeurs. Éditions Nouveaux horizons - ARS : Paris.

Banner JM, Cannon HC. 2011a. L'Art d'Apprendre. Éditions Nouveaux Horizons - ARS : Paris.

Banner JM, Cannon HC. 2011b. L'Art d'Enseigner. Éditions Nouveaux Horizons - ARS : Paris. 
BIMSA. 2009. Manger sain, bon et local. Bulletin d'Information de la Mutualité Sociale Agricole, 96(08): 8-11.

Brauer M. 2011. Enseigner à l'Université Conseils Pratiques, Astuces, Méthodes Pédagogiques. Éditions Armand Colin, France.

Céfaï D. 2003. L'enquête de Terrain. Editions la Découverte : France.

Chupin J. 2004. Main basse sur les cantines scolaires. Le Monde de l'Education, 323: 59-61.

Corinne de Berny-Riche. 2009. Les étudiants franciliens en situation de précarité. Contribution au groupe de travail « étudiants pauvres » de la MIPES d'après les enquêtes de l'OVE. IAU îdF les étudiants franciliens en situation de précarité, $32 \mathrm{p}$.

Darvas P, Gao S, Shen Y, Bawany B. 2018. Enseignement supérieur et équité en Afrique subsaharienne Élargir l'opportunité au-delà de l'élite. Publication du Groupe de Banque Mondiale, suisse, $135 \mathrm{p}$.

Décret $\mathrm{N}^{\circ}$ 686/PR/PM/MESRI/2016 du 01 octobre 2016 portant suppression des bourses internes accordées aux étudiants à compter de l'année académique 20162017. Tchad infos, Tchad.

Degnon GR, Konfo TRC, Adjou SE, Ahoussi ED. 2018. Evaluation des conditions de production, de la qualité physicochimique et microbiologique des cossettes de manioc (Manihot esculanta Crantz) dans la commune de Bassila (Nord-Bénin). Int. J. Biol. Chem. Sci., 12(3): 1528-1541. DOI: https://dx.doi.org/10.4314/ijbcs.v12i3.36

Delamaire C. 2002. De l'alimentation à la santé. Dossier Santé de l'Homme, 358: 15-44.

Diarra CD, Konaté Y, Collin F. 2012. Stratégies d'amélioration de l'offre alimentaire dans une vingtaine d'établissements scolaires de
Sabalibougou en commune V du District de Bamako-MALI. Mémoire de Master en Santé publique et environnement/Intervention en promotion de la santé, École de Santé Publique de l'Université Henri Poincaré de Nancy, Nancy, 76 p.

Djibrine MA, Tidjani A, Ngandolo BN, Nadlaou B and Barro N. 2018. Microbiological quality of some street foods in N'Djamena, Chad: case of sandwiches. Int. J. Biol. Chem. Sci., 12(3): $1113-1122$. DOI: https://dx.doi.org/10.4314/ijbcs.v12i3.3

Felouzis G. 2000. Repenser les inégalités à l'université. Des inégalités sociales aux inégalités locales dans trois disciplines universitaires. Sociétés Contemporaines, (38): 67-97.

Flenon JA, Mitchikpe CES, Hounhouigan DJ. 2018. Facteurs socioéconomiques et de mode de vie associés à l'obésité chez les enfants d'âge scolaire fréquentant les écoles primaires privées de Cotonou. Int. J. Biol. Chem. Sci., 12(1): 217-232. DOI : https://dx.doi.org/10.4314/ijbcs.v12i1.17

Kassel E, Le Roux Y. 2020. Étude comparative de systèmes alimentaires de restauration collective publique en Région Grand Est, Mai 2020. ENSAIA, Université de Lorraine, Lorraine, $111 \mathrm{p}$.

Laaser U. 2020. The Interdependence of Society, Environment and Health and Its Relevance for Societal Development. EURAS Journal of Health, 1(1): 1-19. DOI:10.17932/EJOH.2020.022/ejoh_v01 i1001

Lafay L, Volatier J-L, Martin A. 2002. La restauration scolaire dans l'enquête INCA. Associations entre facteurs sociodémographiques, mode de vie et fréquentation de la restauration scolaire ( $1^{\text {ère }}$ partie). Cahiers de Nutrition et de Diététique, 37(1): 36-44.

Lambert-Le MM, Morlaix S. 2012. La performance académique des étudiants en 
première année universitaire : influence des capacités cognitives et de la motivation. Thèse de Doctorat en Sciences de l'éducation, Université de Bourgogne, Bourgogne, $326 \mathrm{p}$.

Levresse E. 2009. Cantines : des progrès insuffisants. Education Magazine, 2: 1623.

Loukou AL, Anvoh KYB, Kouakou KH, Brou K. Nutritional composition and bioavailability prediction calcium, iron, zinc and magnesium in Justicia galeopsis leaves in Côte d'Ivoire. Int. J. Biol. Chem. Sci., 12(6): 2615-2625. DOI: https://dx.doi.org/10.4314/ijbcs.v12i6.12

Marope M, Gallagher C, Griffin P. 2017. Transformer l'enseignement, l'apprentissage, et l'évaluation, un changement de paradigme global. IBE UNESCO, International Bureau of Education, $25 \mathrm{p}$.

Martin A. 2005. La place de la nutrition dans les enseignements scolaires. Cahiers de Nutrition et de Diététique, 40(2): 81-86.

Morlaix S, Suchaut B. 2012. Analyse de la réussite en première année universitaire : effets des facteurs sociaux, scolaires et cognitifs. Les documents de travail de l'IREDU, Tchad, $12 \mathrm{p}$.

Ndouyang CJ, Ridine W, Yadang KR, Manta R. 2020. Releasing factors of diabetes mellitus. Journal of Medical Research and Health Sciences, 3(7): 1021-1028. DOI:

https://doi.org/10.15520/jmrhs.v6i7.209
Paivandi S, Espinosa G. 2013. Les TIC et la relation entre enseignants et étudiants à l'Université. Distances et Médiations des Savoirs, 3. DOI: 10.4000/dms.425

Paivandi S. 2012. L'appréciation de l'environnement d'études et la manière d'étudier des étudiants. Mesure et Evaluation en Education, 35(3):145-173.

Pavaut B, Vieux F, Darmon N. 2011. Le Resto'U : une aide pour respecter le PNNS? Cahiers de Nutrition et de Diététique, 46: 21-29.

Peraya D. 2011. Un regard sur la « distance», vue de la «présence ». Distances et Savoirs, 3(9): 445-452.

Pinel J-P. 2003. Malaise dans la transmission : l'Université au défi des mutations culturelles contemporaines. Connexions, $78: 11-30$.

Sahouegnon HH, Mitchikpe EC, Kayode APP, Dossa RAM. 2014. Contribution du manioc à l'alimentation et à la nutrition des enfants dans la commune de Djidja au Bénin. Int. J. Biol. Chem. Sci., 8(4): 17571770.

DOI: http://dx.doi.org/10.4314/ijbcs.v8i4.34

Vuarchere P, Guye O, Argaud D. 2006. La restauration en milieu scolaire : état des lieux en région Rhône-Alpes. Cahiers de Nutrition et de Diététique, 41(3): 171179.

WFP (World Food programme). 2019. L'analyse coût-bénéfice de l'alimentation scolaire à Madagascar. WFP, Suisse, 36p. 